\section{Hemorragia pulmonar por leptospirosis}

\section{Pulmonary hemorrhage due to leptospirosis}

El síndrome de hemorragia pulmonar o alveolar difusa (HPD) es un cuadro clínico caracterizado por hemoptisis, disminución de la hemoglobina y/o hematocrito e infiltrados pulmonares bilaterales en la radiografía de tórax que expresa la ocupación alveolar por sangre ${ }^{1,2}$. Si bien la historia clínica, las determinaciones serológicas e inmunológicas y el examen anatomopatológico permiten aproximarse al diagnóstico en la mayoría de los casos, otras causas de HPD no suelen ser tenidas en cuenta inicialmente, lo que trae aparejado serias consecuencias pronósticas ${ }^{2,3}$. La HPD por leptospirosis es una de ellas ${ }^{2-4}$.

Presentamos el caso de un paciente de sexo masculino de 23 años que consulta por un cuadro de 5 días de astenia, fiebre, mialgias y diarrea acuosa. Como antecedente personal sólo destacaba su ocupación esporádica como trabajador en una perrera en la localidad de Vigo, Pontevedra. Al examen físico se objetivó fiebre e ictericia, y en la analítica, leucocitosis $(12.000 / \mu \mathrm{l})$, anemia $(\mathrm{Hb}, 11 \mathrm{mg} / \mathrm{dl})$, plaquetopenia $(53.000 / \mu \mathrm{l})$, aumento de creatincinasa (CPK, $2.400 \mathrm{UI} / \mathrm{l})$, alteración de la función renal (creatinina, $2,3 \mathrm{mg} / \mathrm{dl}$ ), hipertransaminasemia leve e hiperbilirrubinemia mixta (bilirrubina total, 4,33 mg/dl). La radiografía de tórax mostró un patrón alveolointersticial bilateral (fig. 1). Con sospecha de neumonía atípica, HPD por leptospirosis o síndrome pulmón-riñón, se lo ingresó y se inició tratamiento con ceftriaxona y claritromicina y se solicitaron pruebas serológicas e inmunológicas al laboratorio. Durante las primeras 12 horas, el paciente presentó aumento de la expectoración hemoptoica, progresión de los infiltrados alveolares (fig. 2) y baja saturación de oxígeno (presión arterial de oxígeno de $52 \mathrm{mmHg}$ y saturación del $79 \%$ con fracción inspiratoria de oxígeno de 0,5 ) y la analítica de control mostró profundización de la anemia $(\mathrm{Hb}$,

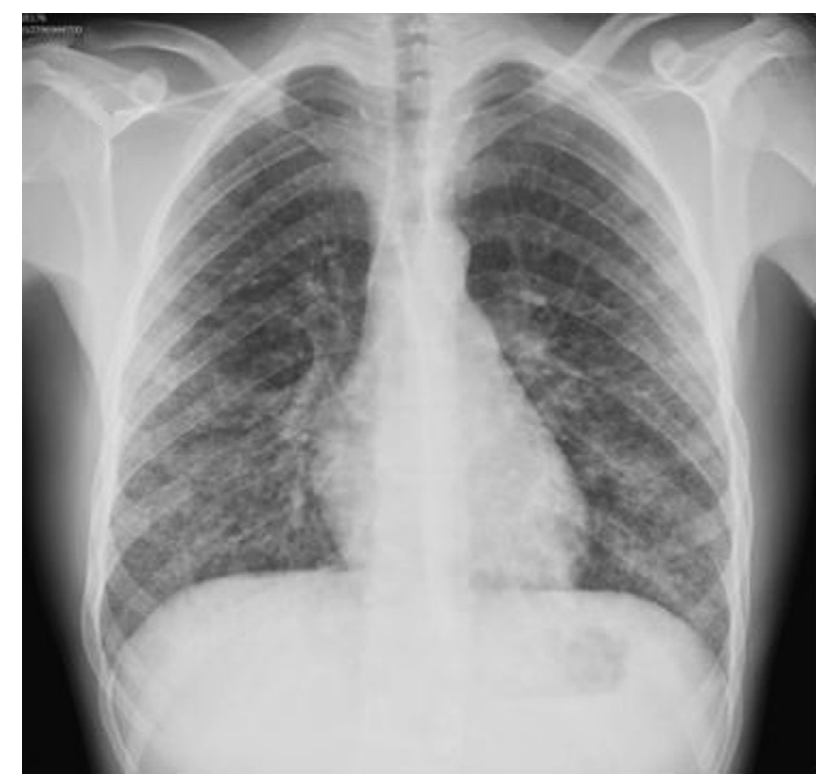

Figura 1 Radiografía de tórax al ingreso que muestra un infiltrado alveolointersticial bilateral.

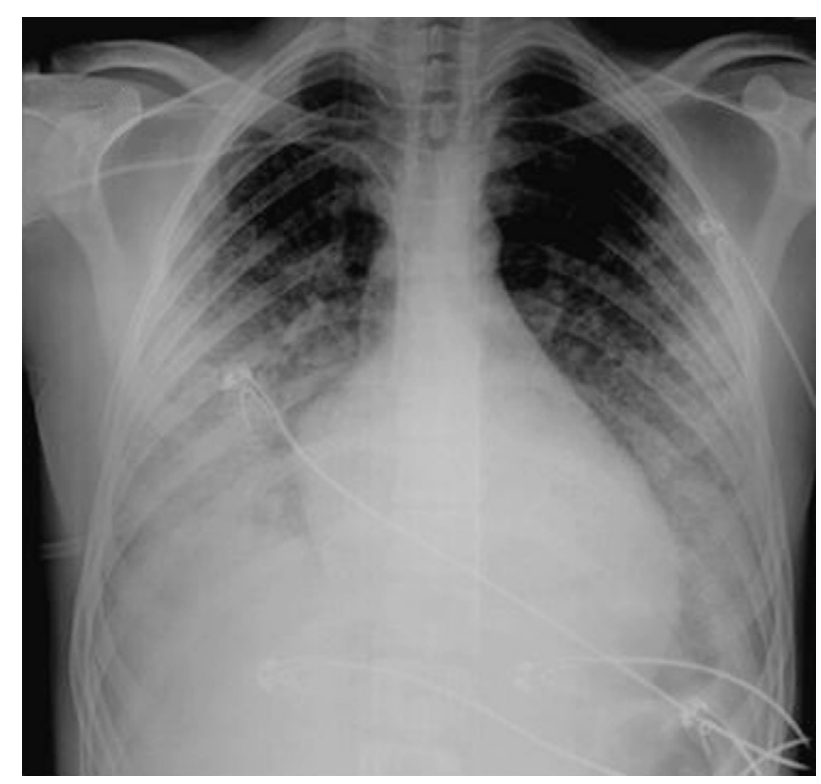

Figura 2 Radiografía de tórax 12 horas después que muestra progresión de los infiltrados alveolares bilaterales predominantemente en campos medios e inferiores.

$6,4 \mathrm{mg} / \mathrm{dl})$ y empeoramiento de la creatinina $(4,6 \mathrm{mg} / \mathrm{dl})$. Se decidió su control en la unidad de cuidados intensivos y al segundo día requirió intubación orotraqueal y ventilación mecánica con fracción inspirada de oxígeno de 1 . Ese mismo día se recibió la serología positiva para $L$. interrogans (IgM, 1:128); el resto de los estudios serológicos e inmunológicos resultaron negativos. Se agregó tratamiento con metilprednisolona a $1 \mathrm{mg} / \mathrm{kg}$. Los días posteriores presentó varios episodios de hemoptisis con repercusión hemodinámica, requirió lavados bronquioloalveolares diarios y terapia con óxido nítrico. Al noveno día el paciente falleció por fallo multiorgánico.

La leptospirosis (LP) es una zoonosis reemergente de amplia distribución mundial producida por Leptospira interrogans. La expresión clínica es muy variada; la mayoría es subclínica o de curso leve ${ }^{5}$. Cuando el cuadro es florido, se describen dos tipos de enfermedad. La forma anictérica que presenta una primera fase, o leptospirémica, caracterizada por fiebre, mialgias intensas, cefalea, inyección conjuntival de 4 a 9 días de duración y que da paso, después de 3 días sin síntomas, a una segunda fase, o inmune, de 3 a 5 días donde reaparecen los síntomas constitucionales y la fiebre junto a otras expresiones clínicas, por ejemplo, meningitis aséptica. La forma ictericohemorrágica o enfermedad de Weil es la presentación clínica menos frecuente (5-10\%), pero más grave y se caracteriza por el desarrollo de ictericia, insuficiencia renal aguda y diátesis hemorrágica en la fase inmune ${ }^{5}$.

La afección pulmonar puede ser variable en incidencia, entre el 20 y el $70 \%$ según las comunicaciones, y severidad $^{3-6}$. Lo habitual es un compromiso leve o moderado en el contexto clínico de una enfermedad dominada por síntomas extrapulmonares ${ }^{4-6}$. Menos frecuentemente (10-15\%), la aparición de disnea e insuficiencia respiratoria asociada a la presencia de infiltrados alveolares bilaterales progresivos y disminución del hematocrito, en presencia o no de hemoptisis, propone el diagnóstico de HPD, manifestación 
más grave del compromiso pulmonar y que se asocia a una mortalidad elevada $(60-70 \%)$, sobre todo cuando se asocia a síndrome de distrés respiratorio agudo ${ }^{3,4}$.

La hemorragia alveolar difusa por leptospirosis suele aparecer en las formas graves de la enfermedad en el contexto de ictericia, nefropatía aguda, trombocitopenia o diátesis hemorrágica, aunque puede ocurrir en la forma anictérica, lo que dificulta claramente el diagnóstico ${ }^{5,7}$. El hallazgo anatomopatológico característico de este cuadro es una vasculitis de pequeños vasos (vasculitis capilar) (2,3,7 $^{2}$ La menor cantidad de leptospiras en el tejido pulmonar en relación con lo que ocurre en otros órganos como el hígado o riñón, junto a la presencia de material antigénico leptospirósico granular en las células del endotelio capilar demostrado por inmunohistoquímica, hace suponer que la lesión vascular pulmonar está gatillada por toxinas ${ }^{3,7}$. Por otro lado, algunos estudios involucran predominantemente al sistema inmunitario en la patogenia de la lesión pulmonar ${ }^{8-10}$. La severidad de la hemorragia pulmonar y del daño renal parece estar en relación directa con la intensidad de la respuesta inmunitaria humoral, valorada por las concentraciones de lgG específicas (los pacientes con mayor repercusión clínica presentaban valores superiores de $\lg G)^{8}$. Asimismo, la demostración del depósito de IgG, IgA y C3 en la membrana basal alveolar, similar a lo que ocurre en el síndrome de Goodpasture, junto a la presencia relativamente escasa de leptospiras, señala que estas últimas podrían precipitar un proceso autoinmunitario exagerado que lleva a la hemorragia pulmonar por daño de los septos alveolares 9 . Por último, la buena respuesta al tratamiento con bolos de metilprednisolona (500 o $1.000 \mathrm{mg}$ ) o ciclofosfamida en casos de HPD publicada en algunas series reafirma la importancia de este mecanismo $0^{9,10}$.

La leptospirosis no suele ser tenida en cuenta cuando abordamos a un paciente con HPD o con un síndrome renopulmonar. Los cambios climáticos que han ocurrido en los últimos años han favorecido el desarrollo de inundaciones en países con climas templados, incluida España. Las inundaciones y las condiciones precarias de urbanización son los principales factores de riesgo ambientales para la transmisión de la enfermedad ${ }^{5}$. Es por esto que consideramos importante que, ante un paciente con HPD, se incluya la letospirosis entre los diagnósticos diferenciales, sobre todo teniendo en cuenta que los pacientes pueden beneficiarse del tratamiento inmunosupresor agresivo.

\section{Bibliografía}

1. Martín Suárez I, Escalera Zalvide A. Protocolo diagnóstico de la hemorragia pulmonar. Medicine. 2009;10:2096-9.

2. Gomez-Roman JJ. Hemorragias alveolares difusas pulmonares. Arch Bronconeumol. 2008;44:428-36.

3. Luks AM, Lakshminarayanan S, Hirschmann JJ. Leptospirosis presenting as diffuse alveolar hemorrhage: case report and literature review. Chest. 2003;123:639-43.

4. Pamplona E, Ribeiro Carvalho CR. Pulmonary leptospirosis. Curr Opin Pulm Med. 2000;6:436-41.

5. Levett PN. Leptospirosis. Clin Microbiol Rev. 2001;14:296-326.

6. Marquez-Martín E, Valera-Bestard B, Luque-Marquez R, Alarcón-Gonzalez A. Afectación pulmonar en la leptospirosis. Arch Bronconeumol. 2006;42:202-4.

7. Dolhnikoff M, Mauad T, Bethlem EP, Carvalho CRR. Pathology and pathophysiology of pulmonary manifestations in leptospirosis. Braz J Inf Dis. 2007;11:142-8.

8. Nally JE, Chantranuwat C, Wu XY, Fishbein MC, Pereira MM, Da Silva JJ, et al. Alveolar septal deposition of immunoglobulin and complement parallels pulmonary hemorrhage in a guinea pig model of severe pulmonary leptospirosis. Am J Pathol. 2004;164:1115-27.

9. Trivedi SV, Chavda RK, Wadia PZ, Sheth V, Baghade PN, Trivedi SP, et al. Role of glucocorticoid pulse therapy in pulmonary involvement in leptospirosis. J Assoc Physicians India. 2001;49:901-3.

10. Trivedi SV, Vasava AH, Patel TC, Bhatia LC. Cyclophosphamide in pulmonary alveolar hemorrhage due to leptospirosis. Indian J Crit Care Med. 2009;13:79-84.

J. Montero-Tinnirello ${ }^{\mathrm{a}, *}$, J. de la Fuente-Aguado ${ }^{\mathrm{b}}$, M. Ochoa-Diez ${ }^{\mathrm{c}}$ y R. Cabadas-Avión ${ }^{\mathrm{C}}$

a Servicio de Medicina Intensiva, Hospital Povisa, Vigo, Pontevedra, España

b Servicio de Medicina Interna, Hospital Povisa, Vigo, Pontevedra, España

c Servicio de Medicina Intensiva, Departamento de Anestesiología y Reanimación, Hospital Povisa, Vigo, Pontevedra, España

* Autor para correspondencia.

Correo electrónico: jjmmmontero@hotmail.com

(J. Montero-Tinnirello).

doi:10.1016/j.medin.2011.04.002

\section{Fascitis necrosante tipo I tras eventroplastia y cierre de ileostomía}

\section{Type I necrotizing fasciitis following eventroplasty and ileostomy}

Varón de 55 años con un índice de masa corporal de 43, hipertenso, con enfermedad obstructiva crónica, insuficiencia cardiaca congestiva y fibrilación auricular. En tratamiento con amiodarona, acenocumarol, furosemida, bromuro de ipratropio y terbutalina. Presenta como antecedentes quirúrgicos una endarterectomía femoral derecha y la rea- lización de una hemicolectomía derecha 6 meses antes por adenocarcinoma. Presentó al sexto día postoperatorio una fuga anastomótica, la cual fue tratada mediante rafia de la dehiscencia puntiforme e ileostomía lateral de protección. Durante el postoperatorio tardío se apreció fístula enterocutánea de bajo débito resuelta de forma conservadora.

El paciente acudió a nuestro servicio para la reconstrucción del tránsito y reparación de eventración gigante.

Se procedió al cierre de ileostomía lateral y reparación de la hernia ventral de $10 \mathrm{~cm}$ mediante eventroplastia tipo Chevrel con malla de polipropileno.

Al sexto día postoperatorio se apreciaron datos clínicos de alarma; hipotensión, taquicardia y fiebre. En la explora- 